Internet Engineering Task Force (IETF)

Request for Comments: 6336

Updates: 5245

Category: Standards Track

ISSN : 2070-1721
M. Westerlund

Ericsson

C. Perkins

University of Glasgow

July 2011

IANA Registry for Interactive Connectivity Establishment (ICE) Options

Abstract

It has been identified that "Interactive Connectivity Establishment (ICE) : A Protocol for Network Address Translator (NAT) Traversal for Offer/Answer Protocols" (RFC 5245) is missing a registry for ICE options. This document defines this missing IANA registry and updates RFC 5245 .

Status of This Memo

This is an Internet Standards Track document.

This document is a product of the Internet Engineering Task Force (IETF). It represents the consensus of the IETF community. It has received public review and has been approved for publication by the Internet Engineering Steering Group (IESG). Further information on Internet Standards is available in section 2 of RFC 5741.

Information about the current status of this document, any errata, and how to provide feedback on it may be obtained at http://www.rfc-editor.org/info/rfc6336.

Copyright Notice

Copyright (c) 2011 IETF Trust and the persons identified as the document authors. All rights reserved.

This document is subject to BCP 78 and the IETF Trust's Legal Provisions Relating to IETF Documents

(http://trustee.ietf.org/license-info) in effect on the date of publication of this document. Please review these documents carefully, as they describe your rights and restrictions with respect to this document. Code Components extracted from this document must include Simplified BSD License text as described in section $4 . e$ of the Trust Legal Provisions and are provided without warranty as described in the Simplified BSD License. 
Table of Contents

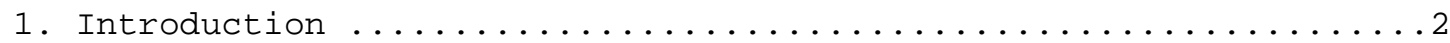

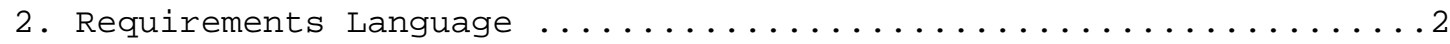

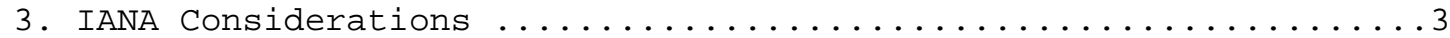

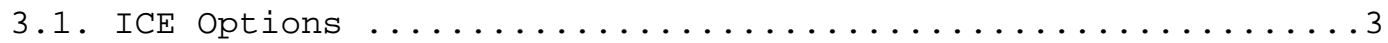

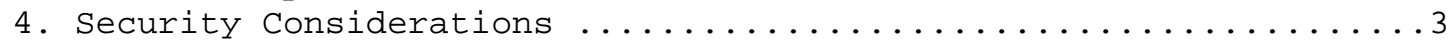

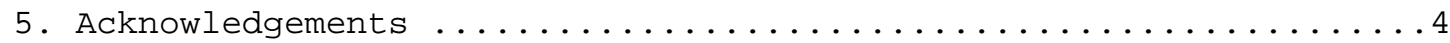

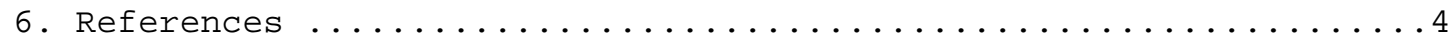

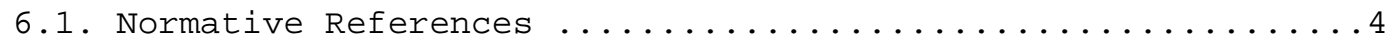

6.2. Informative References ......................

1. Introduction

"Interactive Connectivity Establishment (ICE): A Protocol for Network Address Translator (NAT) Traversal for Offer/Answer Protocols" [RFC5245] defines a concept of ICE options. However, the ICE RFC fails to create an IANA registry for ICE options. As one ICE option is under specification in [ECN-FOR-RTP], there is now a need to create the registry.

RFC 5245 says: "ICE provides for extensibility by allowing an offer or answer to contain a series of tokens that identify the ICE extensions used by that agent. If an agent supports an ICE extension, it MUST include the token defined for that extension in the ice-options attribute".

Thus, as future extensions are defined, these ICE options need to be registered with IANA to ensure non-conflicting identification. The ICE option identifiers are used in signalling between the ICE endpoints to negotiate extension support. RFC 5245 defines one method of signalling these ICE options, using the Session Description Protocol (SDP) with Offer/Answer [RFC3264].

This document updates the ICE specification [RFC5245] to define the "Interactive Connectivity Establishment (ICE) Options" registry.

\section{Requirements Language}

The key words "MUST", "MUST NOT", "REQUIRED", "SHALL", "SHALL NOT", "SHOULD", "SHOULD NOT", "RECOMMENDED", "MAY", and "OPTIONAL" in this document are to be interpreted as described in RFC 2119 [RFC2119]. 


\section{IANA Considerations}

This document defines a registry "Interactive Connectivity Establishment (ICE) Options" for ICE options that can be used in the SDP "ice-options" attribute or other signalling parameters carrying the ICE options.

\subsection{ICE Options}

An ICE option identifier MUST fulfill the ABNF [RFC5234] syntax for "ice-option-tag" as specified in [RFC5245]. This syntax is reproduced here for simplicity, but the authoritative definition is in the ICE RFC:

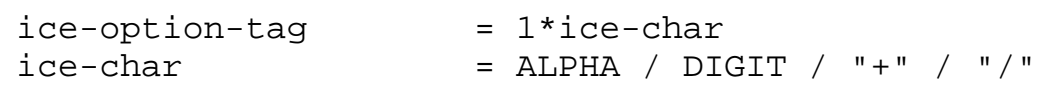

This document registers no ICE option.

4. Security Considerations

As this document defines an IANA registry for an already existing concept, there are no new security considerations. 


\section{Acknowledgements}

The authors would like to thank the people who reviewed the document and provided feedback: Flemming Andreasen, Mykyta Yevstifeyev, Amanda Baber, and Brian Carpenter.

6. References

6.1. Normative References

[RFC2119] Bradner, S., "Key words for use in RFCs to Indicate Requirement Levels", BCP 14, RFC 2119, March 1997.

[RFC5226] Narten, T. and H. Alvestrand, "Guidelines for Writing an IANA Considerations section in RFCs", BCP 26, RFC 5226, May 2008 .

[RFC5234] Crocker, D., Ed., and P. Overell, "Augmented BNF for Syntax Specifications: ABNF", STD 68, RFC 5234, January 2008 .

[RFC5245] Rosenberg, J., "Interactive Connectivity Establishment (ICE) : A Protocol for Network Address Translator (NAT) Traversal for Offer/Answer Protocols", RFC 5245, April 2010.

6.2. Informative References

[ECN-FOR-RTP ]

Westerlund, M., Johansson, I., Perkins, C., O'Hanlon, P., and K. Carlberg, "Explicit Congestion Notification (ECN) for RTP over UDP", Work in Progress, July 2011.

[RFC3264] Rosenberg, J. and H. Schulzrinne, "An Offer/Answer Model with Session Description Protocol (SDP)", RFC 3264 , June 2002 . 


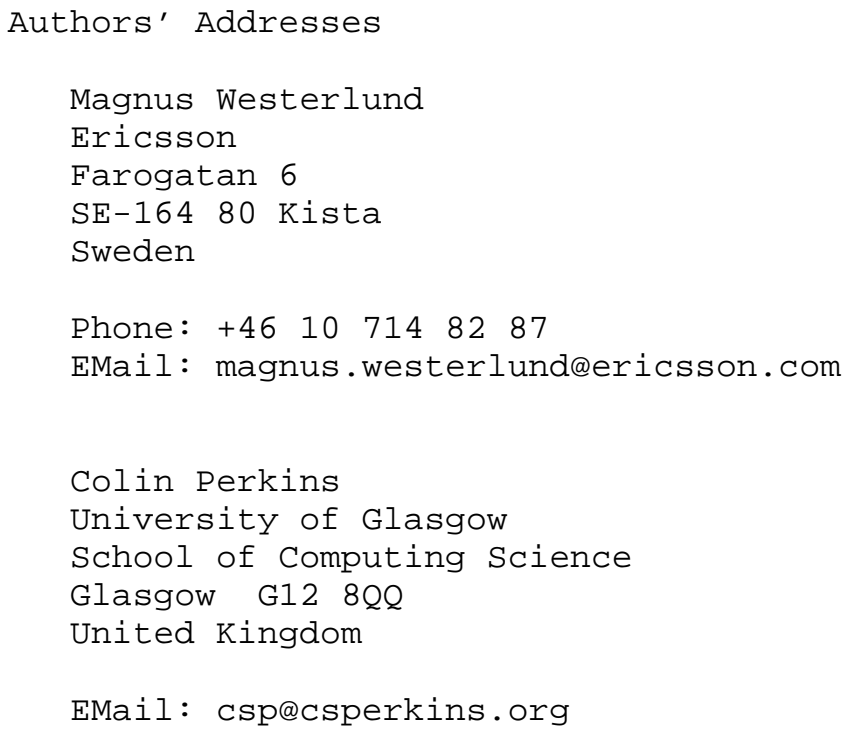

